\title{
The surface and inner temperatures of magnetars
}

\author{
Z. F. Gao ${ }^{1}$, N. Wang ${ }^{1}$ and Q. H. Peng ${ }^{2}$ \\ ${ }^{1}$ Xinjiang Astronomical Observatory, CAS, 150, Science 1-Street, \\ Urumqi Xinjiang, 830011, China \\ email: zhifu_gao@xao.ac.cn \\ ${ }^{2}$ Shchool of Astronomy and Space Science, Nanjing University, \\ Nanjing Jiangshu, 210093, China \\ email: qhpeng@nju.edu.cn
}

\begin{abstract}
Assuming that the timescale of the magnetic field decay is approximately equal to that of the stellar cooling via neutrino emission, we obtain a one-to-one relationship between the effective surface thermal temperature and the inner temperature. The ratio of the effective neutrino luminosity to the effective X-ray luminosity decreases with decaying magnetic field.
\end{abstract}

Keywords. Magnetar, neutrino luminosity, surface thermal temperature, inner temperature.

\section{Introduction}

Magnetars are ultra-magnetized neutron stars (NSs) with magnetic fields largely in excess of the quantum critical field. The majority of magnetars are classified into two populations: the soft gamma-ray repeaters (SGRs), and the anomalous X-ray pulsars (AXPs). Pulsars have been recognized to be normal neutron stars, but sometimes have been argued to be quark stars (e.g., Xu 2005; Du et al. 2009). After their formation, magnetars cool much more efficiently by interior neutrino emission than by surface photon emission. The neutrino emission mechanisms in the stellar cores may be divided into two groups, which leads to standard or rapid cooling. The standard cooling goes mainly via the modified Urca process and the nucleon-nucleon bremsstrahlung process (e.g., Yakovlev \& Pethick 2004), whereas rapid cooling is strongly enhanced by the direct Urca process.

In this paper, we focus on the non-thermal neutrino energy losses in the cores which control cooling of young and middle age $\left(t \leqslant 10^{4}\right.$ yrs, and $B \sim 10^{14}-10^{15} \mathrm{G}$ ) magnetars. In our model, for simplicity, we restrict ourselves by consideration of magnetars whose cores contain the standard composition of dense matter (neutrons, with some admixtures of protons and electrons). In the central region of a magnetar, the electron capture process is expected to occur because of high value of the electron Fermi energy (e.g., Gao et al. 2011a; Gao et al. 2011b). We calculate the effective neutrino luminosity, and simulate numerically the relationship between the surface thermal temperature and the inner temperature of a magnetar.

\section{Surface temperatures of magnetars}

In this section, what we care about is the effective soft X-ray/gamma-ray luminosity $L_{\mathrm{X}}^{\mathrm{eff}}$ and the effective surface temperature $T_{\text {suf }}^{\mathrm{eff}}$ of a magnetar. These two qualities are 


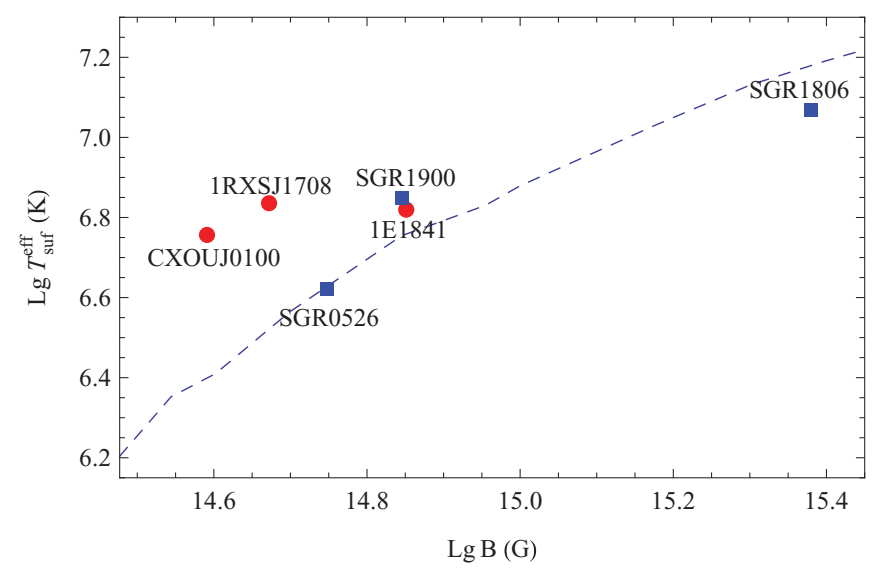

Figure 1. The effective surface thermal temperature $T_{\mathrm{suf}}^{\mathrm{eff}}$ as a function of $B$. Circles and squares represent AXPs and SGRS, respectively.

measured in a local magnetar reference frame. The effective surface temperature is defined by the Stefan law,

$$
L_{\gamma}^{\mathrm{eff}} \simeq L_{\mathrm{X}}^{\mathrm{eff}}=4 \pi R^{2} \sigma\left(T_{\mathrm{suf}}^{\mathrm{eff}}\right)^{4}
$$

where $R$ is the circumferential stellar radius, $\sigma$ is the Stefan-Boltzmann constant, and $L_{\gamma}^{\mathrm{eff}}$ is the thermal surface luminosity in a local magnetar reference frame. By using Eq. 1, we plot the diagram of $L g T_{\text {suf }}^{\text {eff }}$ vs. $L g B$, as shown in Fig. 1.

\section{Inner temperatures of magnetars}

According to our magnetar model, we can compute the effective neutrino luminosity of a magnetar as follows:

$$
\begin{aligned}
& L_{\nu}^{\mathrm{eff}}=\Lambda(B, T) V\left({ }^{3} P_{2}\right) \times \frac{(2 \pi)^{4}}{\hbar V_{1}} G_{F}^{2} C_{V}^{2}\left(1+3 a^{2}\right) \\
& \times \int d^{3} n_{\mathrm{e}} d^{3} n_{\mathrm{p}} d^{3} n_{\mathrm{n}} d^{3} n_{\nu} \delta\left(E_{\nu}+Q-E_{\mathrm{e}}\right) \delta^{3}\left(\overrightarrow{K_{f}}-\overrightarrow{K_{i}}\right) S\left\langle E_{\nu}\right\rangle,
\end{aligned}
$$

where $\Lambda(B, T)$ is the 'Landau level-superfluid modified factor', $S=f_{\mathrm{e}} f_{\mathrm{p}}\left(1-f_{\mathrm{n}}\right)\left(1-f_{\nu}\right)$ $\left(f(\mathrm{j})\right.$ is the fraction of phase space occupied at energy $\left.E_{\mathrm{j}}\right)$, and the rest terms are defined in our recent papers (e.g., Gao et al. 2011a; Gao et al. 2011b; Gao et al. 2012a; Gao et al. $2012 \mathrm{~b})$. We calculate the ratios of $L_{\nu}^{e f f} / L_{\mathrm{X}}^{e f f}\left(\right.$ or $\left.L_{\nu}^{\infty} / L_{\mathrm{X}}^{\infty}\right)$ in different intense fields. The main results are presented as follows: When the magnetic field $B \sim\left(3.0 \times 10^{15}-2.0 \times 10^{14}\right)$ G, accordingly, $L_{\nu}^{\text {eff }} / L_{\mathrm{X}}^{\text {eff }}\left(\right.$ or $\left.L_{\nu}^{\infty} / L_{\mathrm{X}}^{\infty}\right) \sim 22.93 \sim 1.61$. The details can be seen in Fig. 2 . Assuming that the timescale of the magnetic field decay is equal to the the timescale of stellar cooling via neutrino emission, we obtain a one-to-one relationship between $T_{\text {suf }}^{\text {eff }}$ and $T_{\text {int }}^{\text {eff }}$, as shown in Fig. 3.

\section{Conclusions}

Calculations show that $T_{\mathrm{int}}^{\mathrm{eff}}$ is 1-2 orders of magnitude higher than $T_{\text {suf }}^{\mathrm{eff}}$, and the ratio of the magnetar neutrino luminosity to the magnetar soft X-ray luminosity, decreases with decaying magnetic field. 


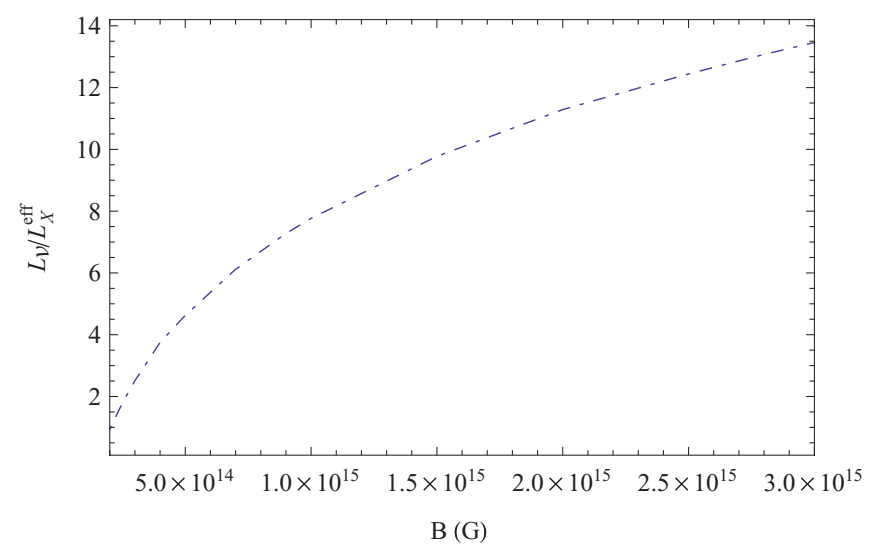

Figure 2. The schematic diagram of $L_{\nu}^{\mathrm{eff}} / L_{\mathrm{X}}^{\mathrm{eff}}\left(\right.$ or $\left.L_{\nu}^{\infty} / L_{\mathrm{X}}^{\infty}\right)$ as a function of $B$.

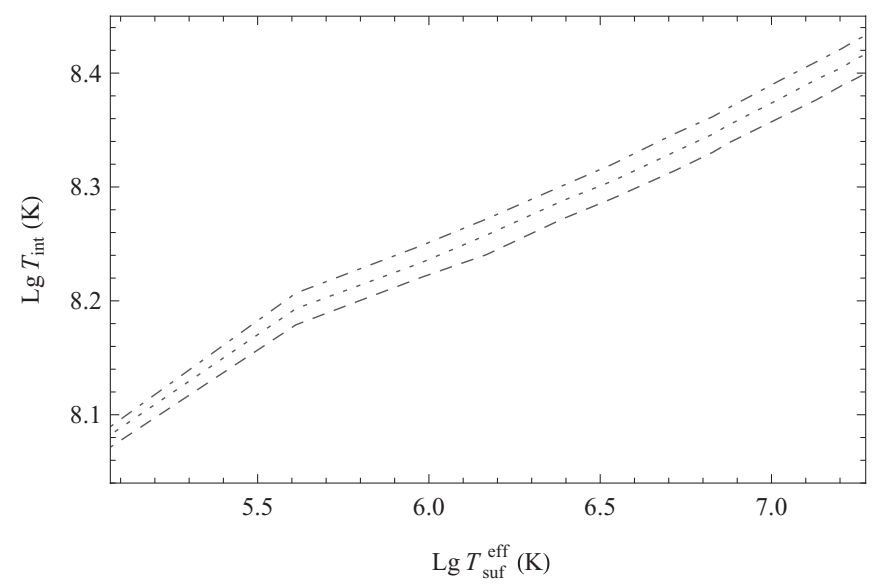

Figure 3. The schematic diagrams of $T_{\mathrm{int}}^{\mathrm{eff}}$ vs. $T_{\mathrm{suf}}^{\mathrm{eff}}$. The range of $T_{\mathrm{suf}}^{\mathrm{eff}}$ is assumed to be $\left(1.835 \times 10^{7} \sim 1.180 \times 10^{5}\right) \mathrm{K}$ arbitrarily, corresponding to $B \sim\left(3.0 \times 10^{15} \sim 1.6 \times 10^{14}\right)$ G. Dot-dashed line, dotted line and dashed line are for the initial value of $T_{\mathrm{int}}^{\mathrm{eff}}=2.70 \times 10^{8} \mathrm{~K}$, $2.60 \times 10^{8} \mathrm{~K}$ and $2.50 \times 10^{8} \mathrm{~K}$, respectively.

\section{Acknowledgements}

This work is partly supported by Chinese National Science Foundation through grant No.10773005, China Ministry of Science and Technology under State Key Development Program for Basic Research (2012CB821800), Knowledge Innovation Program of CAS KJCX2-YW -T09, the Key Directional Project of CAS and NSFC under projects 10173020, 10673021, 10773005, 10778631 and 10903019.

\section{References}

Du, Y. J., Xu, R. X., Qiao, G. J., \& Han, J. L. 2009, MNRAS, 399, 1587

Gao, Z. F., Wang, N., Yuan, J. P., Jiang, L., \& Song, D. L.2011, ApSS, 332, 129

Gao, Z. F., Peng, Q. H., Wang, N., Chou, C.-K., \& Huo, W. S.2011, ApSS, 336, 427

Gao, Z. F., Peng, Q. H., Wang, N., \& Chou, C.-K.2012, Chinese Physics B, Vol. 21. 5, 57109

Gao, Z. F., Peng, Q. H., Wang, N., \& Chou, C.-K. 2012, ApSS, 342, 55

$\mathrm{Xu}$, R. X. 2005, MNRAS, 356, 359

Yakovlev, D. G. \& Pethick, C. J. 2004, ARAA 42, 169 